\title{
Ovarian hyperstimulation syndrome after GnRH agonist trigger and rhCG luteal rescue protocol
}

\author{
Radu Maftei ${ }^{1,2}$, Gabriela Simionescu ${ }^{1,2}$, Sabina Andra Neculai-Valeanu ${ }^{*}, 2$, Edgar \\ Mocanu $^{3}$, Bogdan Doroftei ${ }^{1,2,4}$
}

1"Cuza Voda" University Hospital of Obstetrics and Gynecology, Department of ObstetricsGynecology, Iasi, Romania; ${ }^{2}$ Origyn Fertility Center, Human Reproduction, Iasi, Romania; ${ }^{3}$ Rotunda Hospital, Dublin, Ireland; “"Grigore T. Popa” University of Medicine and Pharmacy lasi, Romania.

\begin{abstract}
Ovarian hyperstimulation syndrome (OHSS) is a rare, but serious iatrogenic complication of In Vitro Fertilization treatments. Since 1943, when the first cases of OHSS were reported, strategies to prevent it have not been very successful. At present, the most used strategy to prevent OHSS is the "freeze-all strategy", which consists in the utilization of a gonadotropin releasing hormone $(\mathrm{GnRH})$ agonist trigger for oocytes maturation in a $\mathrm{GnRH}$ antagonist protocol, followed by cryopreservation of all embryos. In this paper, we present a case of a young woman who developed OHSS after GnRH agonist trigger followed by rhCG luteal rescue protocol. By reporting this case, we want to highlight the importance of OHSS prevention and patients' selection for hCG luteal rescue protocol.
\end{abstract}

Keywords: OHSS, IVF, GnRH agonist, freeze-all, luteal rescue

\section{Introduction}

Ovarian hyperstimulation syndrome (OHSS) is a rare but serious and potentially life-threatening iatrogenic complication of In Vitro Fertilization (IVF) cycles, which occurs in approximately $1-14 \%$ of assisted reproductive technology (ART) cycles [1, 2]. The OHSS is typically associated with exogenous gonadotrophin stimulation and is only rarely observed with use of other agents (clomiphene citrate [CC] and gonadotrophin-releasing hormone [GnRH]) [3].

Pathophysiology of this syndrome is still a subject of debate, the main mechanism is the release of vasoactive peptides from the

Received: October 2016; Accepted after review: December 2016; Published: December 2016.

${ }^{*}$ Corresponding author: Sabina Andra NeculaiValeanu, $\mathrm{PhD}$, Origyn Fertility Center, Human Reproduction, 7D Palat Str, BI. C2, A/1, lasi, Romania.

E-mail: sabina.valeanu@gmail.com granulosa cells causing an increase of vascular permeability. The ultimate OHSS trigger is human chorionic gonadotrophin (hCG) which induces the overexpression of vascular endothelial growth factor (VEGF) in granulosa cells and a rise in serum VEGF concentrations $[4,5]$.

Because of its homology with luteinizing hormone (LH), in IVF treatments, hCG is the most used trigger for final oocyte maturation, and due to its long half-life, in some patients hCG trigger can cause OHSS. Recent good clinical practice recommends that in patients at high risk of OHSS a short GnRH antagonist protocol with $\mathrm{GnRH}$ agonist trigger for final oocytes maturation and "freeze-all strategy" proves the safest.

The most used classification of OHSS is the Golan et al. system [6] (Table I). 


\section{Case report}

We present a case of a 24 year-old woman with history of primary infertility related to male factor (severe asthenozoospermia, WHO 2010) who underwent a procedure of IVF in a center from Romania and presented at our hospital with symptoms of early severe OHSS. The patient BMI was 20.2, the menstrual periods were regular, at 28 days, here AMH was $3.51 \mathrm{ng} / \mathrm{mL}$. The treatment was carried out with $\mathrm{GnRH}$ antagonist downregulated protocol using cetrorelix (Cetrotide; Merck Serono, UK).

Table I. Classification of OHSS based on the clinical manifestations [6]

\begin{tabular}{|c|c|c|}
\hline Form & Degree & Clinical manifestation \\
\hline \multirow[b]{2}{*}{ Mild } & 1 & Abdominal distention and discomfort \\
\hline & 2 & $\begin{array}{l}\text { Criteria of grade } 1+\text { nausea, vomiting, and/or diarrhea. } \\
\text { Ovaries enlarged } 5-12 \mathrm{~cm}\end{array}$ \\
\hline Moderate & 3 & Criteria of mild OHSS + echographic signs of ascites \\
\hline \multirow{3}{*}{ Severe } & 4 & $\begin{array}{l}\text { Criteria of moderate OHSS + clinical signs of ascites and/or hydrothorax } \\
\text { and respiratory distress }\end{array}$ \\
\hline & 5 & $\begin{array}{l}\text { All of the above + changes in blood volume and viscosity, } \\
\text { hemoconcentration, coagulation disorders and decreased renal output and } \\
\text { function }\end{array}$ \\
\hline & 6 & Life-threatening form \\
\hline
\end{tabular}

Ovarian stimulation was started on the second day of menstruation with a daily dose of $125 \mathrm{IU}$ of $\mathrm{rFSH}$ (Puregon; MSD, Oss) until the $7^{\text {th }}$ day of stimulation, afterwards, the stimulation continued with a combination of 50 IU of rFSH (Puregon; MSD, Organon) and 75 IU of hMG (Menopur; Ferring, UK ).

On the $6^{\text {th }}$ day of stimulation, when the leading follicle diameter was $14 \mathrm{~mm}$, the $\mathrm{GnRH}$ antagonist was started. The oestradiol concentration on the day of trigger (day 10) was $10267.7 \mathrm{pmol} / \mathrm{L}$. Final oocyte maturation was triggered by administration of $0.3 \mathrm{mg}$ triptorelin (Diphereline, Ipsen).

After 36 hours a total of 18 oocytes were retrieved. IVF was carried out for all the oocytes and 14 embryos were obtained (fertilization rate: $77.7 \%$ ). On day 5 , two embryos were transferred and 6 embryos were frozen. During oocyte pick up, $125 \mathrm{mcg}$ of recombinant hCG (choriogonadotrophin alfa; Ovitrelle, Serono) was administered and another $125 \mathrm{mcg}$ in the day of embryo transfer. From the day of embryo transfer, 2 $\mathrm{mg} / \mathrm{day}$ of oestradiol valerate (CycloProgynova, Bayer) and $600 \mathrm{mg} /$ day of progesterone (Utrogestan, Laboratories
Besins International) were started. The day following embryo transfer, the patient was admitted to the emergency department of our hospital with moderate abdominal pain, abdominal distention (abdominal circumference $80 \mathrm{~cm}$ ), nausea, vomiting and oliguria. Ultrasound evaluation revealed enlarged ovaries (left ovary: $8.7 \mathrm{~cm}$ and right ovary: $7.9 \mathrm{~cm}$ ) and free fluid in the pouch of Douglas. Laboratory results indicated hemoconcetration: hemoglobin: $17.7 \mathrm{~g} / \mathrm{dl}$; hematocrit: $52 \%$, white blood cells count $31800 \mu \mathrm{L}$ and $\mathrm{K}: 5.31 \mathrm{mmol} / \mathrm{L}$.

The treatment was initiated immediately with albumin $(25 \%)$ in doses of $50 \mathrm{~g}$ infused over $4 \mathrm{~h}$ and repeated at every $12 \mathrm{~h}$ in the first 2 days, lactated ringers solution (1000 $\mathrm{mL} /$ day) in the first 2 days and low molecular weight heparin (Clexane; 6000 IU daily) during hospitalization.

The patient was discharged after 10 days of hospitalization, pregnancy being confirmed biochemically ( $\beta$ hCG on day 10 after embryo transfer was $37 \mathrm{mIU} / \mathrm{mL}$ ). After two weeks, twin pregnancy was confirmed by ultrasound.

At 34 weeks of gestation, due to uterine contraction and fetal distress, she delivered by 
caesarean-section one girl (weight $1900 \mathrm{~g}$, APGAR score 9) and a boy (weight $1700 \mathrm{~g}$, APGAR score 9).

\section{Discussions}

We present here a case of OHSS resulting from luteal phase use of hCG and highlight the danger of pursuing such protocols in the circumstances of a patient at high risk of OHSS. The in vitro fertilization therapy was developed as a way to help infertile couples who are otherwise unable to conceive a child but, unfortunately, IVF is not free of side effects and complications.

The most serious complication of controlled ovarian hyperstimulation cycles is the development of OHSS. The trigger of OHSS is represented by hCG, which determine the overexpression of vascular endothelial growth factor (VEGF) in granulosa cells and raises in serum VEGF concentrations and can have different grades of severity. The incidence of severe OHSS, which can be life-threatening, is 2-3\% [7].

The risk factors for OHSS can be divided in two groups: primary (factors that can be identified before the initiation of stimulation) and secondary (factors that becomes obviously during stimulation). In general, patients at risk to develop OHSS are patients with $\mathrm{AMH}$ levels above $3.3 \mathrm{ng} / \mathrm{dL}[8,9]$, age below 30 years, low BMI, polycystic ovaries syndrome (PCOS), previous OHSS, E2 levels more than $3000 \mathrm{pg} / \mathrm{mL}$ in the day of trigger, rapidly rising of oestradiol levels during stimulation and number of oocyte collected $>15$. Depending on the onset, OHSS can be late (after more than 10 days after trigger) which is primarily related to the ongoing pregnancy and early OHSS, which is an iatrogenic complication of IVF (in the first 10 days after trigger) caused by luteotrophic effect of hCG.

The patient described here had both types of risk factors for OHSS: primary $(\mathrm{AMH}>3.3$ $\mathrm{ng} / \mathrm{dL}$, age $<30$ years, low BMI) and secondary (rapidly rising of oestradiol levels during stimulation: $2856 / 6086 / 10267 \mathrm{pmol} / \mathrm{L}$ in day $5 / 8 / 11$, number of oocyte collected more than
15 [10]) therefore, the $\mathrm{GnRH}$ agonist triggering was mandatory.

In such cases should an embryo transfer take place? It has been proven that where a patient is identified as a high risk, the use of an antagonist protocol with $\mathrm{GnRH}$ analogue trigger prevents early OHSS [11]. The only protocol to prevent late OHSS is adopting a "freeze all" procedure with cryopreservation of all embryos obtained.

The proponents of embryo transfer in these high risk cases use the so called "luteal rescue" protocol with hCG to correct the luteal defect. The most used protocol is administration of $1500 \mathrm{IU}$ of hCG at $35 \mathrm{~h}$ after trigger [12-14]. In this case, a higher dose, 125 $\mathrm{mcg}$ of $\mathrm{rhCG}$ which is equivalent of $3250 \mathrm{IU}$ of hCG, were used, on two occasions. Furthermore, two embryos were transferred, resulting in a twin pregnancy and a more severe form of OHSS as well as premature delivery.

\section{Conclusions}

This case of an early onset of severe OHSS after GnRH agonist triggering combined with rhCG luteal rescue protocol illustrates the significant risks encountered when long acting hCG is offered to women at high risk. The clinical and ethical debate regarding embryo transfer in these cases must have patient's interests first. Is the risk worth taking? Considering that the patient has achieved a delivery of two healthy children, the purists will say, yes! Yet, as OHSS is a potentially fatal condition caution must be considered before transfer and especially in relation to the use of hCG for luteal support in these cases. We consider the use of hCG a dangerous procedure.

We recommend that all ART centers develop guidelines with strict criteria when embryo transfer is performed based on local experience. While we acknowledge that it is difficult to establish what patients are at risk of developing late OHSS after transfer, in the interest of patient safety and good clinical practice a "freeze all" protocol should be routine where a patient has already deemed at high risk of OHSS. We also strongly 
recommend that luteal supplementation with hCG should not be used.

Additionally, we must highlight the fact that in IVF procedures, the most important concerns should be the safety and the rights of the patient [15], and of course, the delivery of one healthy baby, considering the fact that multiple pregnancies are an adverse outcome of assisted reproductive technologies [16, 17].

\section{References}

1. Nastri CO, Ferriani RA, Rocha IA, Martins WP. Ovarian hyperstimulation syndrome: pathophysiology and prevention. $J$ Assist Reprod Genet 2010; 27(2-3):121-128.

2. Youssef MA, Van Wely M, Hassan MA, et al. Can dopamine agonists reduce the incidence and severity of OHSS in IVF/ICSI treatment cycles? A systematic review and metaanalysis. Hum Reprod Update 2010; 16(5):459466.

3. The Practice Committee of American Society for Reproductive Medicine. Ovarian hypersimulation syndrome. Fertil Steril 2008; 90(5):188-193.

4. Neulen J, Yan Z, Raczek S, et al. Human chorionic gonadotropin-dependent expression of vascular endothelial growth factor/vascular permeability factor in human granulosa cells: importance in ovarian hyperstimulation syndrome. J Clin Endocrinol Metab 1995; 80(6):1967-71.

5. Pellicer A, Albert C, Mercader A, BonillaMusoles F, Remohi J, Simon C. The pathogenesis of ovarian hyperstimulation syndrome: in vivo studies investigating the role of interleukin-1 beta, interleukin-6, and vascular endothelial growth factor. Fertil Steril 1999; 71(3):482-9.

6. Golan A, Ron-el R, Herman A, Soffer $Y$, Wienraub Z, Caspi E. Ovarian hyperstimulation syndrome: an update review. Obstet Gynecol Surv 1989; 44(6):430-440.

7. Papanikolaou EG, Pozzobon C, Kolibianakis $\mathrm{EM}$, et al. Incidence and prediction of ovarian hyperstimulation syndrome in woman undergoing gonadotropin-releasing hormone antagonist in vitro fertilization cycles. Fertil Steril 2006; 85(1):112-120.

8. Ocal P, Sahmay S, Cetin M, Irez T, Guralp O, Cepni I. Serum anti-Mullerian hormone and antral follicle count as predictive markers of OHSS in ART cycles. J Assist Reprod Genet 2011; 28(12):1197-1203.

9. Lee $\mathrm{TH}$, Liu $\mathrm{CH}$, Huang CC, et al. Serum antiMullerian hormone and estradiol levels as predictors of ovarian hyperstimulation

syndrome in assisted reproduction technology cycles. Hum Reprod 2008; 23(1):160-167.

10. Steward RG, Lan $L$, Shah $A A$, et al. Oocyte number as a predictor for ovarian hyperstimulation syndrome and live birth: an analysis of 256,381 in vitro fertilization cycles. Fertil Steril 2014; 101(4):967-973.

11. Engmann L, DiLuigi $A$, Schmidt $D$, Nulsen J, Maier D, Benadiva $\mathrm{C}$. The use of gonadotropinreleasing hormone $(\mathrm{GnRH})$ agonist to induce oocyte maturation after cotreatment with $\mathrm{GnRH}$ antagonist in high-risk patients undergoing in vitro fertilization prevents the risk of ovarian hyperstimulation syndrome: a prospective randomized controlled study. Fertil Steril 2008; 89(1):84-91.

12. Radesic B, Tremellen K. Oocyte maturation employing a $\mathrm{GnRH}$ agonist in combination with low-dose hCG luteal rescue minimizes the severity of ovarian hyperstimulation syndrome while maintaining excellent pregnancy rates. Hum Reprod 2011; 26(12):3437-3442.

13. Seyhan A, Ata B, Polat M, Son WY, Yarali H, Dahan MH. Severe early ovarian hyperstimulation syndrome following $\mathrm{GnRH}$ agonist trigger with the addition of $1500 \mathrm{IU}$ hCG. Hum Reprod 2013; 28(9):2522-2528.

14. Humaidan $P$, Ejdrup Bredkjaer $\mathrm{H}$, Westergaard LG, Yding Andersen C. 1,500 IU human chorionic gonadotropin administered at oocyte retrieval rescues the luteal phase when gonadotropin-releasing hormone agonist is used for ovulation induction: a prospective, randomized, controlled study. Fertil Steril 2010; 93(3):847-854.

15. Crisan $O$, Speranta I. Patients' rights in pharmacy legislation. Farmacia 2014; 62(3):444-450.

16. van Wely $M$, Twisk $M$, Mol BW, van der Veen $\mathrm{F}$. Is twin pregnancy necessarily an adverse outcome of assisted reproductive technologies? Hum Reprod 2006; 21(11):2736-2738.

17. Belaisch-Allart $\mathrm{J}$. Is twin pregnancy necessarily an adverse outcome of assisted reproductive technologies? Hum Reprod 2007; 22:1495. 